\title{
Efficient synthesis of L-lactic acid from glycerol by metabolically engineered Escherichia coli
}

\author{
Suman Mazumdar ${ }^{1 \dagger}$, Matthew D Blankschien ${ }^{1 \dagger}$, James M Clomburg ${ }^{1}$ and Ramon Gonzalez ${ }^{1,2^{*}}$
}

\begin{abstract}
Background: Due to its abundance and low-price, glycerol has become an attractive carbon source for the industrial production of value-added fuels and chemicals. This work reports the engineering of $E$. coli for the efficient conversion of glycerol into L-lactic acid (L-lactate).

Results: Escherichia coli strains have previously been metabolically engineered for the microaerobic production of D-lactic acid from glycerol in defined media by disrupting genes that minimize the synthesis of succinate, acetate, and ethanol, and also overexpressing the respiratory route of glycerol dissimilation (GlpK/GlpD). Here, further rounds of rationale design were performed on these strains for the homofermentative production of L-lactate, not normally produced in E. coli. Specifically, L-lactate production was enabled by: 1), replacing the native D-lactate specific dehydrogenase with Streptococcus bovis L-lactate dehydrogenase (L-LDH), 2) blocking the methylglyoxal bypass pathways to avoid the synthesis of a racemic mixture of D- and L-lactate and prevent the accumulation of toxic intermediate, methylglyoxal, and 3) the native aerobic L-lactate dehydrogenase was blocked to prevent the undesired utilization of L-lactate. The engineered strain produced $50 \mathrm{~g} / \mathrm{L}$ of L-lactate from $56 \mathrm{~g} / \mathrm{L}$ of crude glycerol at a yield $93 \%$ of the theoretical maximum and with high optical (99.9\%) and chemical (97\%) purity.

Conclusions: This study demonstrates the efficient conversion of glycerol to L-lactate, a microbial process that had not been reported in the literature prior to our work. The engineered biocatalysts produced L-lactate from crude glycerol in defined minimal salts medium at high chemical and optical purity.
\end{abstract}

Keywords: L-lactic acid, Glycerol, Metabolic engineering, Escherichia coli

\section{Background}

Glycerol has recently become an inexpensive and abundant carbon source due to being a byproduct of the biodiesel, oleo-chemical, and bioethanol industries, $[1,2]$. In addition, future opportunities are available for even larger amounts of glycerol production due to the synthesis and intracellular accumulation of high glycerol concentrations by certain species of algae [3]. Although many microorganisms are able to metabolize glycerol, the use of industrial microbes such as E. coli could greatly accelerate the development of platforms to produce fuels and chemicals from this carbon source [4]. We recently reported on the ability of E. coli to

\footnotetext{
* Correspondence: Ramon.Gonzalez@rice.edu

${ }^{\dagger}$ Equal contributors

${ }^{1}$ Department of Chemical and Biomolecular Engineering, Rice University, 6100 Main Street, MS-362, Houston, TX 77005, USA

${ }^{2}$ Department of Bioengineering, Rice University, Houston TX, USA
}

metabolize glycerol under anaerobic and microaerobic conditions and identified the pathways mediating these metabolic processes (Figure 1) [5-7]. These studies have provided a platform to metabolically engineer $E$. coli for the efficient conversion of glycerol into fuels and industrial chemicals such as ethanol [8-11], hydrogen [11,12], formic acid [11], pyruvic acid [13] and succinic acid [14].

A shared metabolic feature of the anaerobic and microaerobic utilization of glycerol in $E$. coli is the generation of ethanol as the primary product and the negligible production of lactic acid (lactate) [5-7]. However, we have recently reported the engineering of this bacterium for microaerobic production of D-lactate from glycerol in a defined minimal medium [15]. Lactate and its derivatives have many applications in the food, pharmaceutical, and polymer industries [16,17]. An example is polylactic acid, a renewable, biodegradable, and environmentally friendly polymer produced from controlled ratios of D- and L-lactate [18]. Because 


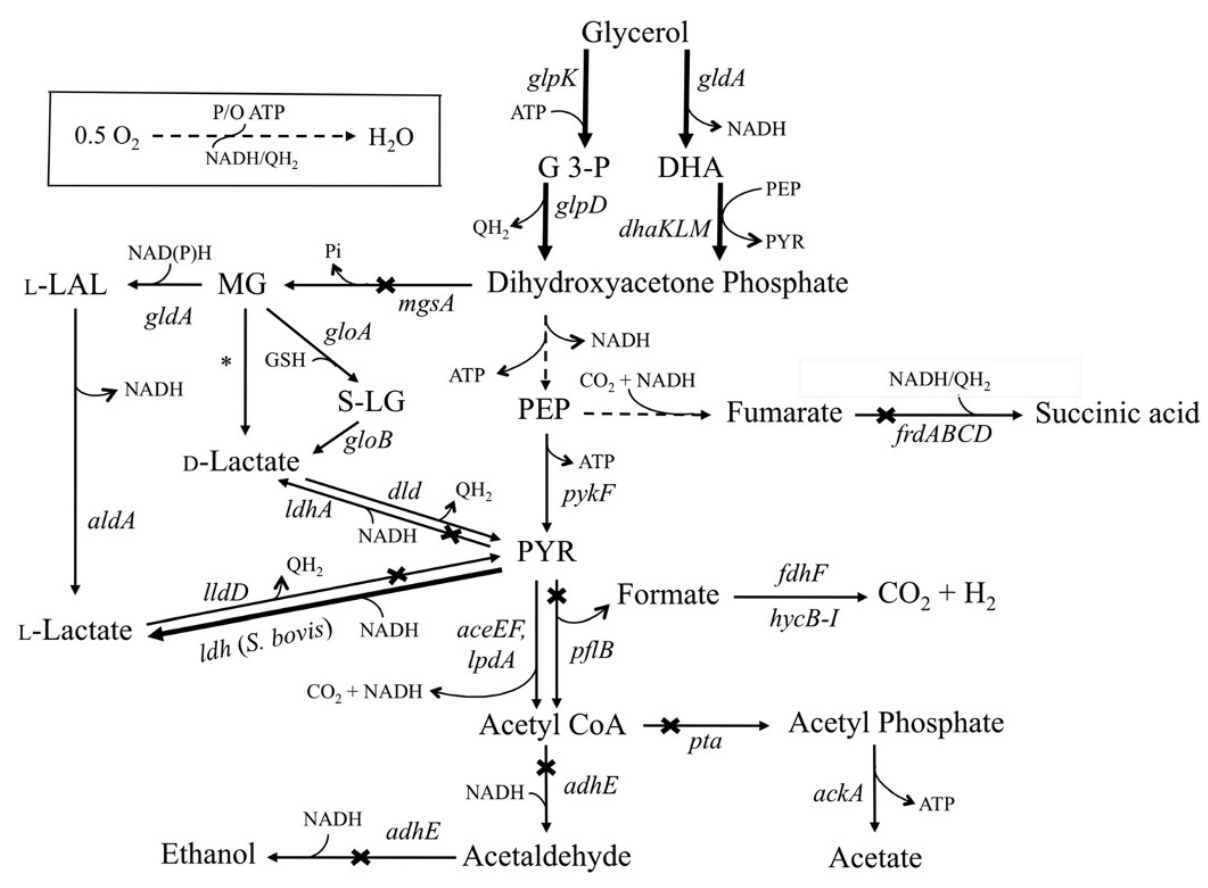

Figure 1 Pathways involved in the microaerobic utilization of glycerol and the synthesis of fermentation products in native and engineered $E$. coli. Genetic modifications supporting the metabolic engineering strategies employed in this work are illustrated by thicker lines (overexpression of E. coli gldA-dhaKLM and glpK-glpD and S. bovis Idh) or cross bars (disruption of pflB, pta, adhE, frdA, IdhA, mgsA and IIdD). Broken lines illustrate multiple steps. Relevant reactions are represented by the names of the gene(s) coding for the corresponding enzymes (E. coli genes/enzymes unless otherwise specified in parenthesis): aceEF-IpdA, pyruvate dehydrogenase complex; adhE, acetaldehyde/alcohol dehydrogenase; ackA, acetate kinase; aldA, aldehyde dehydrogenase $\mathrm{A}$; dhaKLM, dihydroxyacetone kinase; dld, respiratory D-lactate dehydrogenase; $f d h F$, formate dehydrogenase, part of fomate hydrogenlyase complex; FrdABCD, fumarate reductase; gldA, glycerol dehydrogenase; gloA, glyoxalase I; gloB, glyoxalase II; glpD, aerobic glycerol-3-phosphate dehydrogenase; glpK, glycerol kinase; hycB-I, hydrogenase 3, part of formate hydrogenlyase complex; Idh, fermentative L-lactate dehydrogenase (S. bovis); IdhA, fermentative D-lactate dehydrogenase; IIdD, respiratory L-lactate dehydrogenase; $m g s A$, methylglyoxal synthase; $p f(B$, pyruvate formate-lyase; pta, phosphate acetyltransferase; pykF, pyruvate kinase. Abbreviations: DHA, dihydroxyacetone; DHAP, DHA phosphate; G-3-P, glycerol-3-phosphate; PEP, phosphoenolpyruvate; $P_{i}$, inorganic phosphate; PYR, pyruvate; P/O, amount of ATP produced in the oxidative phosphorylation per pair of electrons transferred through the electron transport system; $\mathrm{QH}_{2}$, reduced quinones; S-LG, S-lactoylglutathione; *, glyoxalase III.

of the importance of using pure enantiomers in such applications, biological processes have the advantage over chemical means of producing chirally pure lactate from inexpensive media containing only the carbon source and mineral salts [19]. While lactic acid bacteria have been traditionally used in the production of $\mathrm{D}$ - and L-lactate from carbohydrate-rich feedstocks, several studies have recently reported alternative biocatalysts such as $E$. coli $[16,17]$, many of which are engineered to produce L-lactate from sugar feedstocks [20-23].

Unlike the aforementioned reports (i.e. use of carbohydrates), our laboratory has focused on the use of glycerol as a carbon source for the production of chemicals with high optical and chemical purity. As such, this manuscript focuses on the metabolic engineering of $E$. coli for the efficient conversion of glycerol to L-lactate, a microbial process that had not been reported prior to our work. The engineered strains hold great promise for the conversion of low-value glycerol streams present in the current biofuels industries to a highervalued product, L-lactate.

\section{Results}

Replacement of $E$. coli's D-lactate specific dehydrogenase with Streptococcus bovis L-lactate dehydrogenase and disruption of the methylglyoxal bypass

E. coli strains LA01 ( $\triangle p f l B \Delta f r d A)$ and LA02 ( $\Delta p t a$ $\triangle a d h E \Delta f d A)$ are initial platforms developed to demonstrate the microaerobic production of optically pure D-lactate in mineral salts medium using glycerol [15]. LA01 contains a deletion in pflB (pyruvate formate lyase, PFL), which minimizes the production of ethanol and acetate due to the fact that PFL is the primary route for pyruvate conversion to acetyl-CoA during the microaerobic utilization of glycerol [5]. LA01 also possess an $\operatorname{frd} A$ deletion (component of fumarate reductase) to reduce the synthesis of succinate (Figure 1). LA02, on the other hand, is a triple mutant in which the synthesis of 
ethanol $(\triangle a d h E)$, acetate $(\Delta p t a)$, and fumarate $(\Delta f r d A)$ have been blocked through respective gene deletions directly involved with their synthesis (Figure 1). Both strains produced D-lactate as the primary product of glycerol metabolism (Figure 2A and Table 1, rates of 0.34 and $0.30 \mathrm{~g} / \mathrm{L} / \mathrm{h}$, respectively) under microaerobic conditions (Additional file 1: Figure S1).

To initiate the metabolic engineering of these previous LA01 and LA02 platforms for the production of L-lactate, the fermentative $E$. coli D-lactate dehydrogenase (D-LDH) was eliminated, resulting in strains LA06 (LA01 $\Delta l d h$ A) and LA07 (LA02 $\Delta l d h A)$. As expected, very small amounts of lactate (final titers of $\sim 0.1 \mathrm{~g} /$ liter in both cases) were detected in the fermentation broth of strains LA06 and LA07 (Figure 2A), demonstrating that D-LDH $(l d h A)$ is the primary route of lactate production in these $E$. coli platforms. The lactate produced was a racemic mixture of D- and L-lactate (Figure 3), suggesting their production through the MG detoxification pathways [24-27] (Figure 1).

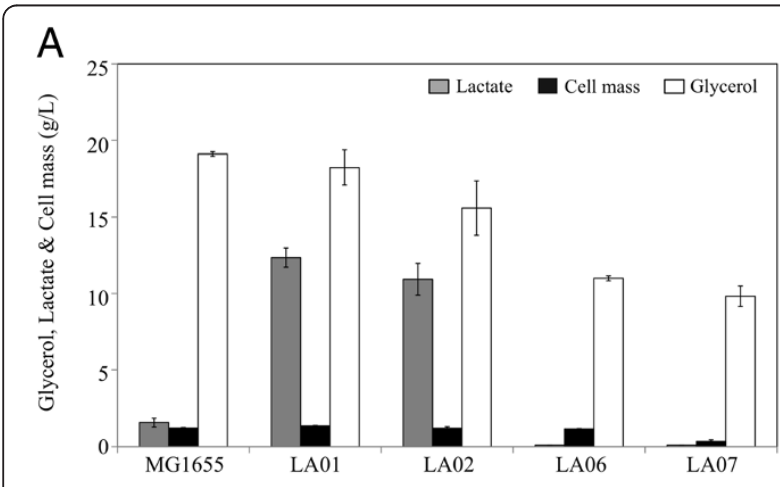

B

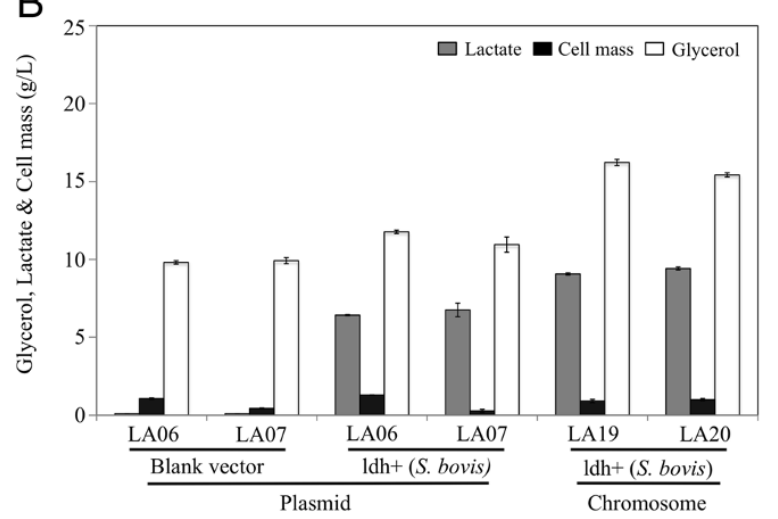

Figure 2 Cell growth, glycerol utilization, and lactate synthesis in 36-hour shake flasks cultures of wild-type MG1655 and engineered strains. (A) LA01 ( $\triangle p f \mid B \triangle f r d A), L A 02$ ( $\Delta p t a \Delta a d h E \Delta f r d A)$, LA06 ( $\triangle p f l B \Delta f r d A \Delta / d h A)$ and LA07 ( $\Delta$ pta $\Delta a d h E \Delta$ frdA $\Delta / d h A$ ). (B) LA06 (pZSblank), LA07 (pZSblank), LA06 (pZSldh), LA07 (pZSIdh), LA19 ( $\Delta p f l B \Delta f r d A \Delta m g s A \Delta / d h A l d h+$ ), and LA20 ( $\Delta$ pta $\Delta a d h E \Delta$ frdA $\Delta m g s A \Delta / d h A l d h+$ ). Gene expressions from either plasmid or chromosomal integration are indicated by a " + " next to the corresponding gene(s) or operon(s). Error bars represent standard deviations for triplicate measurements.
The ability of strains LA06 and LA07 to produce large amounts of lactate was restored by the presence of plasmid pZSIdh which expresses the L-lactate dehydrogenase (L-LDH) from S. bovis (Figure $2 \mathrm{~B}$ and Table 1, rates of $\sim 0.19 \mathrm{~g} / \mathrm{L} / \mathrm{h}$ for both). The enantiomeric purity of the produced L-lactate was high in both cases ( 99.5\%, Figure 3).

Given the above results, the S. bovis $l d h$ gene was chromosomally integrated in strains LA01 and LA02 and the $E$. coli $m g s A$ gene was simultaneously deleted to avoid any production of D-lactate through the MG bypass. The $l d h A$ locus was chosen as the integration site because the levels of expression of D-LDH from this promoter in LA01 and LA02 were shown to support efficient production of D-lactate [15] and could presumably support L-lactate production as well. The resulting LA19 ( $\Delta p f l B \Delta$ frdA $\Delta m g s A \Delta l d h A l d h+)$ and LA20 ( $\triangle p t a \Delta a d h E \Delta f r d A \Delta m g s A \Delta l d h A l d h+)$ strains performed well, producing $\sim 9 \mathrm{~g} /$ liter of L-lactate in 36 hours $(\sim 0.25 \mathrm{~g} / \mathrm{L} / \mathrm{h})$ (Figure $2 \mathrm{~B})$. However, strain LA20 exhibited a slightly better lactate yield $(0.61 \mathrm{~g} / \mathrm{g}$ glycerol compared to $0.56 \mathrm{~g} / \mathrm{g}$ glycerol in LA19) and lower acetate production (discussed below) (Figure 2B and Table 1). As postulated, the expression of $l d h$ from the $l d h A$ promoter resulted in L-LDH activity levels similar to those reported for D-LDH in strains LA01 and LA02: i.e. $0.136 \pm 0.005 \mu \mathrm{mol} / \mathrm{mg}$ protein $/ \mathrm{min}$ in LA20 (Table 2) compared to $0.082 \pm 0.005 \mu \mathrm{mol} / \mathrm{mg}$ protein $/ \mathrm{min}$ in LA01 [15]. Finally, the lactate produced by LA20 was 99.9\% L-lactate, with similar enantiomeric purity found for strain LA19 (Figure 3).

\section{Overexpression of glycerol-utilization and L-lactate synthesis pathways and elimination of the endogenous pathway for L-lactate utilization}

Although strains LA19 and LA20 produced L-lactate at high chemical and chiral purity, the kinetics of glycerol utilization and lactate synthesis, including lactate titer and yield, were inferior to that of the LA01 and LA02 parental strains (compare panels A and B in Figure 2; Table 1). Since we have previously shown that the conversion of glycerol to D-lactate can be accelerated by amplifying either glycerol-utilization or lactatesynthesis pathways [15], we investigated whether similar strategies could be implemented in the production of L-lactate.

Two primary routes can mediate the conversion of glycerol to the common intermediate, dihydroxyacetone (DHAP) under microaerobic conditions [5] (Figure 1). A fermentative pathway converts glycerol to dihydroxyacetone (DHA) via glycerol dehydrogenase ( gldA) and then to DHAP through the action of DHA kinase $($ dhaKLM). The alternative route is a respiratory/aerobic pathway composed of the enzymes glycerol kinase 
Table 1 Glycerol consumption, product synthesis, and carbon recovery in cell mass and fermentation products during the microaerobic utilization of glycerol in minimal medium by wild-type and engineered strains ${ }^{\mathrm{a}}$

\begin{tabular}{|c|c|c|c|c|c|c|c|c|c|}
\hline \multirow{2}{*}{ Strain } & \multirow{2}{*}{$\begin{array}{c}\text { Glycerol } \\
\text { consumed } \\
(g / L)\end{array}$} & \multicolumn{6}{|c|}{ Product synthesized (g/L) } & \multicolumn{2}{|c|}{ Carbon recovery $^{\mathbf{b}}$} \\
\hline & & Acetate & Succinate & Ethanol & Lactate & Pyruvate & Biomass & Products & Overall \\
\hline & & & & & & & & & \\
\hline MG1655 & $19.1(0.1)$ & $2.9(0.1)$ & $2.4(0.0)$ & $2.1(0.1)$ & $1.6(0.3)$ & $0.0(0.0)$ & $1.2(0.0)$ & $67.4(1.4)$ & $75.4(1.6)$ \\
\hline
\end{tabular}

Strains engineered for the production of D-lactate

LA01

$\mathrm{LAO2}$

Strains engineered for the production of L-lactate

\section{$18.2(1.1) \quad 0.6(0.1)$ \\ $15.6(1.8) \quad 0.6(0.0)$}

$0.0(0.0)$

$0.1(0.0)$

$0.0(0.0)$

$0.0(0.0)$

$12.3(0.6)$

$0.0(0.0)$

$0.0(0.0)$

$1.4(0.0)$

$74.9(3.4)$

$84.3(3.7)$

$10.9(1.0)$

ton

$1.2(0.1) \quad 77.6(2.8)$

87.4 (3.4)

\begin{tabular}{|c|c|c|c|c|c|c|c|c|c|}
\hline LA06 & $11.0(0.2)$ & $2.0(0.1)$ & $0.1(0.0)$ & $0.0(0.0)$ & $0.1(0.0)$ & $0.0(0.0)$ & $1.2(0.0)$ & $27.8(0.5)$ & $67.2(0.9)$ \\
\hline LA07 & $9.8(0.7)$ & $1.2(0.4)$ & $0.2(0.1)$ & $0.0(0.0)$ & $0.1(0.0)$ & $0.0(0.0)$ & $0.35(0.1)$ & $20.7(5.5)$ & $34.0(2.2)$ \\
\hline LA06 (pZS) & $9.8(0.1)$ & $1.8(0.1)$ & $0.1(0.0)$ & $0.0(0.0)$ & $0.1(0.0)$ & $0.0(0.0)$ & $1.1(0.1)$ & $29.2(1.6)$ & $70.0(0.1)$ \\
\hline LA07 (pZS) & $9.9(0.2)$ & $1.5(0.0)$ & $0.3(0.0)$ & $0.0(0.0)$ & $0.1(0.0)$ & $0.0(0.0)$ & $0.4(0.0)$ & $30.1(0.6)$ & $46.7(2.0)$ \\
\hline LA06 (pZSIdh) & $11.8(0.1)$ & $0.6(0.0)$ & $0.1(0.0)$ & $0.0(0.0)$ & $6.4(0.0)$ & $0.0(0.0)$ & $1.3(0.0)$ & $64.9(0.2)$ & $78.6(0.2)$ \\
\hline LA07 (pZSIdh) & $10.9(0.5)$ & $0.2(0.0)$ & $0.1(0.0)$ & $0.0(0.0)$ & $6.7(0.4)$ & $0.0(0.0)$ & $0.3(0.1)$ & $65.7(2.9)$ & $70.4(1.9)$ \\
\hline LA19 & $16.2(0.2)$ & $1.0(0.0)$ & $0.0(0.0)$ & $0.0(0.0)$ & $9.1(0.1)$ & $0.0(0.0)$ & $0.9(0.1)$ & $67.4(0.2)$ & $74.5(1.1)$ \\
\hline LA20 & $15.4(0.1)$ & $0.4(0.0)$ & $0.2(0.0)$ & $0.0(0.0)$ & $9.4(0.1)$ & $0.0(0.0)$ & $1.0(0.1)$ & $67.7(0.2)$ & $75.8(0.5)$ \\
\hline LA19 (pZSKLMgldA) & $16.6(0.6)$ & $1.0(0.0)$ & $0.0(0.0)$ & $0.2(0.0)$ & $10.9(0.4)$ & $0.0(0.0)$ & $0.4(0.0)$ & $79.0(0.4)$ & $82.1(0.9)$ \\
\hline LA19 (pZSglpKglpD) & $16.8(0.9)$ & $0.7(0.1)$ & $0.0(0.0)$ & $0.0(0.0)$ & $11.7(1.2)$ & $0.0(0.0)$ & $0.8(0.0)$ & 79.7 (3.9) & $85.9(3.8)$ \\
\hline LA19 (pZSIdh) & $13.2(1.6)$ & $0.6(0.1)$ & $0.0(0.0)$ & $0.0(0.0)$ & $7.4(1.3)$ & $0.0(0.0)$ & $0.7(0.1)$ & $65.6(4.6)$ & $74.4(3.4)$ \\
\hline LA20 (pZSKLMgldA) & $15.2(0.3)$ & $0.4(0.1)$ & $0.1(0.0)$ & $0.0(0.0)$ & $10.1(0.5)$ & $0.0(0.0)$ & $0.9(0.2)$ & $72.9(1.5)$ & $80.3(3.1)$ \\
\hline LA20 (pZSglpKglpD) & $18.6(0.4)$ & $0.6(0.0)$ & $0.2(0.0)$ & $0.0(0.0)$ & $13.7(0.7)$ & $0.0(0.0)$ & $0.8(0.0)$ & $76.4(1.7)$ & $82.8(1.6)$ \\
\hline LA20 (pZSIdh) & $15.3(0.2)$ & $0.2(0.0)$ & $0.2(0.1)$ & $0.0(0.0)$ & $10.0(0.1)$ & $0.0(0.0)$ & $0.9(0.0)$ & $70.5(1.6)$ & $77.6(2.0)$ \\
\hline LA20 (pZSglpK.glpD) & $41.0(0.0)$ & $1.0(0.0)$ & $0.7(0.0)$ & $0.0(0.0)$ & $32.6(0.1)$ & $0.0(0.0)$ & $1.1(0.0)$ & $86.4(0.3)$ & $89.6(0.3)$ \\
\hline LA20 & $41.6(0.0)$ & $0.8(0.0)$ & $0.6(0.0)$ & $0.0(0.0)$ & $34.7(0.0)$ & $0.0(0.0)$ & $1.6(0.0)$ & $90.3(0.0)$ & $95.0(0.1)$ \\
\hline LA20 & $40.4(0.0)$ & $0.9(0.0)$ & $0.9(0.0)$ & $0.0(0.0)$ & $32.8(0.1)$ & $0.0(0.0)$ & $1.1(0.0)$ & $88.4(0.2)$ & $91.9(0.1)$ \\
\hline 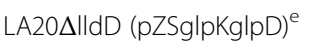 & $57.2(0.0)$ & $1.6(0.0)$ & $1.2(0.0)$ & $0.0(0.0)$ & $50.1(0.0)$ & $0.0(0.0)$ & $2.0(0.0)$ & $95.2(0.1)$ & $99.4(0.7)$ \\
\hline
\end{tabular}

${ }^{a}$ Data represent the average of three samples (standard deviations shown in parenthesis) taken from 36-hour shake flask cultures grown on minimal medium supplemented with $20 \mathrm{~g} / \mathrm{L}$ of glycerol, unless otherwise specified.

b Carbon recovery is expressed as the percent mol of carbon in product, including biomass, per mol of carbon in glycerol consumed. The column "product" shows the total recovery of carbon in products, assuming that moles of acetate plus moles of ethanol equals moles of 1-C compounds (formate plus CO2) generated by the dissimilation of pyruvate. The column "overall" shows the overall carbon recovery, including products and biomass.

c Cultures in which $40 \mathrm{~g} / \mathrm{L}$ of glycerol was used and samples were taken at 72 hours (all glycerol was consumed).

${ }^{\mathrm{d}}$ A culture in which $40 \mathrm{~g} / \mathrm{L}$ of crude glycerol derived from biodiesel production was used and samples were taken at 72 hours (all glycerol was consumed).

${ }^{\mathrm{e}} \mathrm{A}$ culture in which $60 \mathrm{~g} / \mathrm{L}$ of crude glycerol ( $40 \mathrm{~g} / \mathrm{L}$ initially present and $20 \mathrm{~g} / \mathrm{L}$ added at 48 hours) was used and samples were taken at 84 hours ( 57 g/L of glycerol were consumed).

(glpK) and glycerol-3-phosphate (G3P) dehydrogenase $(g l p D)$ which mediates the conversion of glycerol to G3P and subsequently to DHAP, respectively. Overexpression of either one of the pathways in LA19 (0.3 and $0.33 \mathrm{~g} / \mathrm{L} / \mathrm{h}$ for fermentative and respiratory routes, respectively) and LA20 ( 0.28 and $0.38 \mathrm{~g} / \mathrm{L} / \mathrm{h}$ for fermentative and respiratory routes, respectively) led to faster utilization of glycerol and L-lactate synthesis, although the respiratory pathway led to higher L-lactate titers and yields (Table 1, 2). Coupling of glycerol-3-phosphate oxidation and oxygen reduction via the quinine pools $[28,29]$ likely results in the preferential synthesis of L-lactate due to the fact that the overall conversion of glycerol to lactate becomes a redox balanced pathway. In addition, ATP would be generated by both substrate-level phosphorylation and the respiratory chain (see Figure 1 and Discussion).

Another limiting factor for lactate synthesis in strains LA19 and LA20 could be insufficient levels of L-lactate dehydrogenase due to less expression from the chromosomal copy of $S$. bovis $l d h$ as opposed to plasmid overexpression. Thus, expression of $l d h$ from a plasmid could alleviate this limitation and lead to an increase in the fraction of carbon diverted towards the 


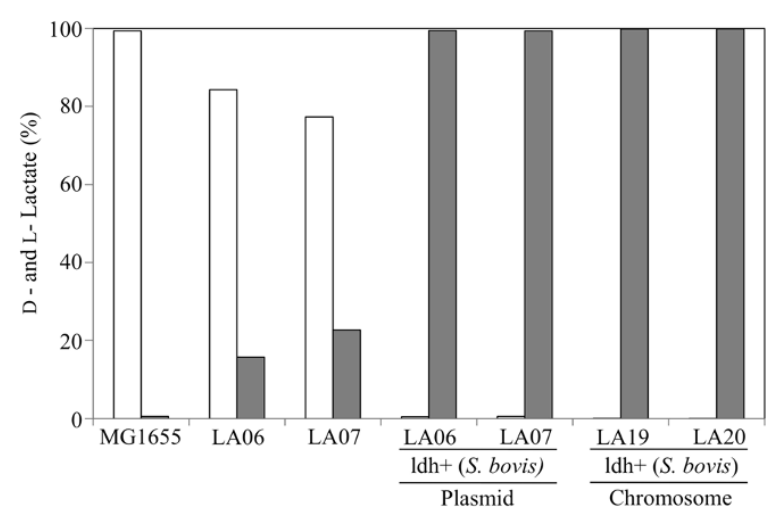

Figure 3 Enantiomeric composition determined enzymatically of lactate produced by wild-type MG1655 and engineered strains LA06, LA07, LA06 (pZSIdh), LA07 (pZSIdh), LA19, and LA20. The percentage of each enantiomer in the mixture is shown: D-lactate (white bar) and L-lactate (gray bar).

synthesis of L-lactate (increasing L-lactate yield) and/or the flux of the glycerol-to- L-lactate pathway (increasing the rate of L-lactate production). This strategy led to a slight increase in the production of L-lactate in LA20 [pZSIdh] (Table 1), which was arguably caused by the 5 -fold increase in the activity of L-LDH (Table 2). In contrast, overexpression of L-LDH had no beneficial effect on lactate production or glycerol utilization in strain LA19 [pZSldh] (Table 1). Thus, plasmid overexpression of S. bovis ldh was not deemed more beneficial than that of the chromosomal copy and not explored further.

Of note, strain LA19 ( $\Delta p f l B \Delta f r d A \Delta m g s A \Delta l d h A l d h+)$ and its parent and derivatives produced much higher concentrations of acetate than that observed in the LA20 ( $\Delta p t a \Delta a d h E \Delta f r d A \Delta m g s A \Delta l d h A l d h+)$ strain and its parent and derivatives (Table 1). While PFL is the primary route for pyruvate conversion to acetyl-CoA during the microaerobic utilization of glycerol, low levels of acetyl-CoA and subsequently acetate could still be formed in the LA19 lineage via leakiness of the primarily aerobic pyruvate dehydrogenase complex
(aceEF and $l p d A$, Figure 1) [5]. As acetate formation in the LA20 lineage is directly blocked by a pta deletion, lower acetate levels would be expected. Increased acetate formation in the LA19 lineage could also explain the differential growth observed between LA06 (i.e. $\Delta p f l B, p t a^{+}$etc.) and LA07 (i.e. $p f l B^{+}, \Delta p t a$ ). As these strains are deleted for endogenous $l d h A$, they cannot readily synthesize any common fermentative product to achieve redox balance and allow continued ATP production. In this context, the small increases in acetate levels seen in the LA06 would be critical for growth as acetate formation results in 2 ATP molecules per glycerol consumed via substrate level phosphorylation (Figure 1). Only when the higher glycerol utilization and subsequent L-lactate synthesis were achieved with the more optimal expression of $S$. bovis ldh from the chromosome (as opposed to from a plasmid) did the growth between the LA19 and LA20 and direct derivatives become similar (Figure 2 and Table 1). As the LA20 lineage was deemed better than that of LA19 and previous work by us has shown no additional benefit of using the $p f l B$ deletion in conjunction with just directly blocking the competing fermentative products (data not shown) we choose to use LA20 as our platform for further metabolic engineering.

Overall, the best performance was observed when the respiratory glycerol-utilization pathway was overexpressed in the LA20 platform (Table 1, and see rates in text above). Using $20 \mathrm{~g} / \mathrm{L}$ of glycerol, LA20 (pZSglpKglpD) produced $13.7 \mathrm{~g} / \mathrm{L}$ of L-lactate $(0.38 \mathrm{~g} / \mathrm{L} / \mathrm{h})$ at a yield of $0.74 \mathrm{~g} \mathrm{~L}$-lactate/g glycerol. Given these results, we further examined the production of L-lactate by LA20 (pZSglpKglpD) in the presence of a higher concentration of glycerol. Starting now with $40 \mathrm{~g} / \mathrm{L}$ of glycerol, this strain produced about $33 \mathrm{~g} /$ liter of L-lactate in less than 72 hours $(0.46 \mathrm{~g} / \mathrm{L} / \mathrm{h})$ at a yield of $0.82 \mathrm{~g}$ L-lactate $/ \mathrm{g}$ glycerol (Figure 4 and Table 1). Besides L-lactate, only small amounts of acetate were found in the culture medium, demonstrating the homolactic nature of the

Table 2 Functional characterization of constructs used in the overexpression of glycerol utilization and L-lactate synthesis enzymes

\begin{tabular}{lll}
\hline & ${\text { Activity }(\boldsymbol{\mu m o l} / \mathbf{m g} \text { protein/min })^{\mathbf{a}}}$ \\
\hline Enzyme tested & LA20 (Control) $)^{\mathrm{b}}$ & LA20 (Overexpressed) $^{c}$ \\
Glycerol kinase & $0.187 \pm 0.005$ & $0.669 \pm 0.004$ \\
Aerobic glycerol-3-phosphate dehydrogenase & $0.017 \pm 0.001$ & $0.027 \pm 0.002$ \\
Glycerol dehydrogenase & $0.049 \pm 0.002$ & $0.39 \pm 0.02$ \\
Dihydroxyacetone kinase & $0.005 \pm 0.001$ & $0.019 \pm 0.002$ \\
L-Lactate dehydrogenase & $0.136 \pm 0.005$ & $0.68 \pm 0.06$ \\
\hline
\end{tabular}

${ }^{a}$ All activities were measured as described in Materials and Methods and values are reported as average \pm standard deviation for triplicate assays.

${ }^{b}$ Activities measured in strain LA20 containing the blank vector.

c Activities measured in strain LA20 containing a plasmid overexpressing the specified enzyme: i.e. pZSKLMgldA for glycerol dehydrogenase and dihydroxyacetone kinase, pZSglpKglpD for glycerol kinase and aerobic glycerol-3-phosphate dehydrogenase, and pZSIdh for S. bovis L-lactate dehydrogenase. Reported values are from 36-hour shake flask cultures. 


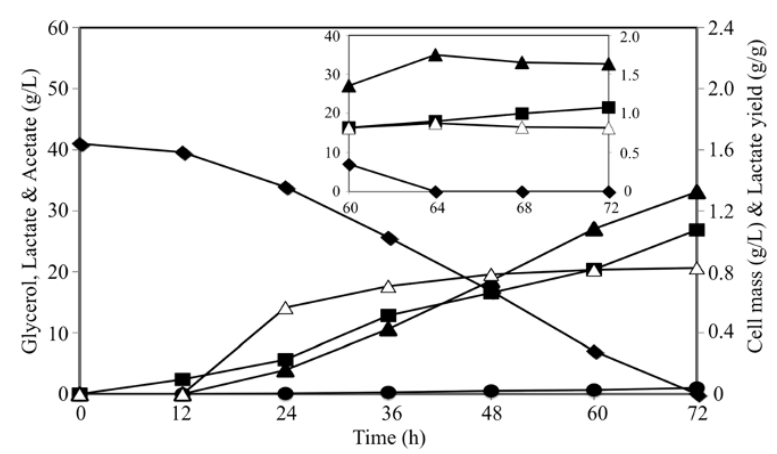

Figure 4 Kinetics of lactate synthesis by strain LA20 (pZSglpKglpD) in shake flasks containing minimal medium with $40 \mathrm{~g} / \mathrm{L}$ of glycerol. Data for concentration of cells ( $)$, glycerol ( $)$, lactate $(\mathbf{\Lambda})$ and acetate $(\bullet)$, along with lactate yield $(\Delta)$, are shown. Coefficients of variation (i.e. standard deviations/average $\times 100$ ) were below $5 \%$ in all cases. The inset shows a high-resolution data set for late stages of cultivation: symbols and axis titles are as specified for the main figure.

fermentation (Table 1). However, a closer examination of the dynamics of cell growth, glycerol consumption and product synthesis at the late stages of the fermentation revealed interesting behavior: the cultures never reached stationary phase, even when all glycerol was consumed, and a decrease in both L-lactate concentration and yield occurred (Figure 4, Inset). Based on these observations, the accumulation of large amounts of L-lactate in the medium was hypothesized to trigger its consumption by the respiratory L-lactate dehydrogenase $(I l d D)$, which can catalyze the oxidation of L-lactate to pyruvate [30]. Deletion of the $l l d D$ gene in strain LA20 and overexpression of the GlpK-GlpD pathway resulted in a clear stationary phase following glycerol depletion from the medium and no decrease in lactate yield or concentration was observed (data not shown). This strain, named LA20 $\Delta l l d D$ (pZSglpKglpD), produced 35.1g/liter of L-lactate from $41.6 \mathrm{~g} /$ liter of glycerol in about 64 hours $(0.55 \mathrm{~g} / \mathrm{L} / \mathrm{h})$ with an overall product yield of $0.86 \mathrm{~g} \mathrm{L-lactate} / \mathrm{g}$ of glycerol, which clearly surpasses the performance of its parent LA20 (pZSglpKglpD) (Table 1).

\section{Production of L-lactate at high concentrations from crude glycerol}

The use of an industrial medium containing crude glycerol generated as a by-product in the biodiesel industry is of great relevance for the biocatalyst developed in this work. Engineered strains performed very well when crude glycerol was used as a carbon source. Glycerol consumption and L-lactate synthesis by strain LA20 $\Delta l l d D$ (pZSglpKglpD) using $40 \mathrm{~g} /$ liter crude glycerol were similar to those reported for the consumption of pure glycerol (Table 1). To better assess the potential of this process, an experiment with even higher concentrations of crude glycerol was conducted. Under these conditions, strain LA20 $\Delta l l d D$ (pZSglpKglpD) produced more than $50 \mathrm{~g} /$ liter of L-lactate in 84 hours at a yield of $0.90 \mathrm{~g} \mathrm{L-lactate} / \mathrm{g}$ glycerol (Figure 5). Maximum and average volumetric rates of L-lactate production of $1.3 \mathrm{~g} / \mathrm{L} / \mathrm{h}$ and $\sim 0.6 \mathrm{~g} /$ liter/h were respectively, achieved.

\section{Discussion}

L-lactate production from sugars can be achieved using native lactic acid bacteria but are constrained by the requirements for complex nutrients and exhibit limitations in both product selectivity and enantiomeric purity $[16,17]$. To overcome these issues, bacteria and yeasts have been engineered to produce L-lactate as the primary product of carbohydrate fermentations [16,17,20-23]. However, the production of L-lactate from glycerol has not been reported. The work conducted here focuses on the metabolic engineering of $E$. coli for the microaerobic production of L-lactate, at high chemical (97\%) and optical (99.9\%) purities, from glycerol in defined minimal salts medium. Using LA20 $\Delta l d d D$ [pZSglpKglpD], $50 \mathrm{~g} /$ liter of L-lactate were produced in 84 hours at a yield of $0.90 \mathrm{~g} \mathrm{L-lactate} / \mathrm{g}$ glycerol (Figure 5) with a yield close to $93 \%$ of the theoretical maximum (0.967 wt/wt) when calculated from equation 2 below. Besides providing a high yield and productivity, the resulting biocatalyst can also utilize crude glycerol as carbon source, which has become an abundant and inexpensive feedstock due to being a by-product of the current biofuel industries [31].

While L-lactate is not a native product of glycerol metabolism in E. coli, its homologous production could

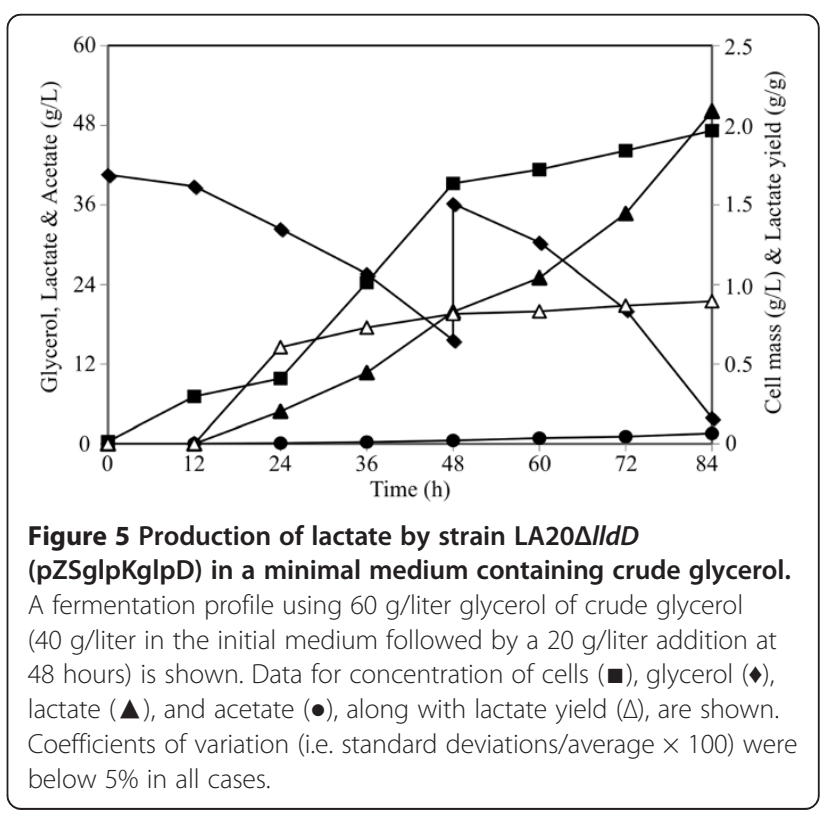


be achieved through a two-step pathway that converts methylglyoxal (MG, an intermediate in the MG bypass) to L-lactaldehyde (L-LAL) and then to L-lactate [24-27] (Figure 1). However, the synthesis of L-lactate through these pathways is not desirable due the existence of several native MG detoxification pathways in E. coli that can lead to the production of both $\mathrm{D}$ - and L-lactate (Figure 1) [25-27], compromising the enantiomeric purity of the product (Figure 3 ). In addition, MG is a very toxic metabolite whose accumulation could severely impair metabolism and lead to cell death $[25,27]$. Finally, the synthesis of L-lactate through the MG route is energy inefficient, as no ATP is generated in the conversion of dihydroxyacetone phosphate (DHAP) to L-lactate (Figure 1). Since the generation of DHAP from glycerol consumes one ATP equivalent (in the form of ATP or PEP), the overall conversion of glycerol to L-lactate through this route would lead to the net consumption of one ATP equivalent per each molecule of L-lactate produced (Figure 1). This route, summarized in the equation 1 below by assuming glycerol dissimilation through the GlpK-GlpD pathway (Figure 1), would also generate one net reducing equivalent per L-lactate synthesized:

$$
\text { Glycerol }+A T P+Q \rightarrow L-\text { lactate }+A D P+P+Q H_{2}
$$

A more attractive alternative to the above MG route that we chose to utilize in this study is the production of L-lactate utilizing enzymes from the later stages of Embden-Meyerhof-Parnas pathway which would avoid the aforementioned disadvantages. In this scenario (see Figure 1), the overall conversion of glycerol to lactate would lead to the net generation of one ATP (via substrate-level phosphorylation) and one reducing equivalent per each molecule of L-lactate produced, as shown in the equation 2 below:

$$
\text { Glycerol }+A D P+P+Q \rightarrow L-\text { lactate }+A T P+Q H_{2}
$$

Since the GlpK-GlpD pathway mediates glycerol dissimilation in the engineered strain and microaerobic conditions were used (see Supplemental Materials and Additional file 1: Figure S1), coupling the overall transfer of electrons from glycerol-3-phosphate to oxygen (combination of GlpD and CyoABCD) [28,29] can be achieved. This coupling could theoretically generate 1.14 ATPs via oxidative phosphorylation per molecule of glycerol dissimilated [32]. However, given the lower experimental values typically observed [33], the synthesis of 1 ATP per glycerol-3-phosphate molecule oxidized is probably a more reasonable assumption and is detailed in equation III below:

$$
\mathrm{QH}_{2}+0.5 \mathrm{O}_{2} \rightarrow \mathrm{H}_{2} \mathrm{O}+\mathrm{ATP}
$$

From equations 2 and 3 it then becomes apparent that the synthesis of L-lactate from glycerol can generate up to two molecules of ATP per molecule of L-lactate produced. Overall, this high ATP yield explains why using the later stages of the Embden-Meyerhof-Parnas pathway with overexpression of the respiratory GlpK-GlpD pathway in LA20 was beneficial (as opposed to the use of the MG route).

Given the beneficial nature of the engineered glycerol-toL-lactate pathway (i.e. redox balanced and ATP generating), we expect that the future use of metabolic evolution approaches will lead to the selection of even more productive biocatalysts. Similar techniques have been successfully implemented in $E$. coli for the efficient production of biofuels and other products [34-36]. Process-based modifications such as fed-batch cultivations and highdensity cultures are also envisioned to further improve the volumetric rates of L-lactate production.

\section{Conclusions}

The present study demonstrates the conversion of glycerol to L-lactate, a microbial process that had not been reported to date prior to this study. The engineered biocatalyst produced L-lactate from glycerol in a defined minimal salts medium at high chemical and optical purity. The high yields and productivities achieved with the use of crude glycerol as carbon source, which has become an abundant and inexpensive feedstock, demonstrate that low-value glycerol streams from the current biofuels industries can be efficiently converted to higher value products such as L-lactate.

\section{Methods}

\section{Strains, plasmids, and genetic methods}

Strains LA01 and LA02 (see Table 3 for genotype) were used as hosts to engineer the production of L-lactate. Gene knockouts were introduced by P1 phage transduction $[11,37]$. Single gene knockout mutants from the National BioResource Project (NIG, Japan) were used as donors of specific mutations [38]. Replacement of native ldhA (encoding D-lactate dehydrogenase) with Streptococcus bovis ldh (encoding L-lactate dehydrogenase) was achieved via a previously reported method for allele replacement using the sacB-containing pWM91 suicide vector [39]. Plasmid pVALDH1 [40], kindly provided by Dr. T. R. Whitehead (National Center for Agricultural Utilization Research, U.S. Department of Agriculture, Agricultural Research Service, Peoria, IL) was the source of the $l d h$ gene and primers c-ldh (Table 3) were used for cloning purposes. All chromosomal disruptions and replacements were confirmed by polymerase chain reaction using the 
Table 3 Strains, plasmids and primers used in this study

\begin{tabular}{|c|c|c|}
\hline $\begin{array}{l}\text { Strain/ Plasmid/ } \\
\text { Primer }\end{array}$ & Description/Genotype/Sequence & Source \\
\hline \multicolumn{3}{|l|}{ Strains $^{a}$} \\
\hline MG1655 & $F-\lambda-i / v G-r f b-50 r p h-1$ & {$[41]$} \\
\hline LA01 & MG1655 $\Delta p$ fIB::FRT $\Delta$ frdA::FRT-Kan-FRT; sequential deletion of pflB and frdA in MG1655 & {$[15]$} \\
\hline LA02 & MG1655 $\Delta$ pta::FRT $\Delta a d h E:: F R T \Delta$ frdA::FRT-Kan-FRT; sequential deletion of pta, adhE and frdA in MG1655 & {$[15]$} \\
\hline LA06 & LA01 $\Delta / d h A:: F R T-K a n-F R T$ & This study \\
\hline LA07 & LA02 $\Delta / d h A:: F R T-K a n-F R T$ & This study \\
\hline LA19 & LA01 $\Delta m g s A:: F R T \Delta / d h A::$ dh & This study \\
\hline LA20 & LA02 $\Delta m g s A:: F R T \Delta / d h A:: I d h$ & This study \\
\hline $\mathrm{LA} 19 \Delta / / d D$ & LA01 $\Delta m g s A:: F R T \Delta / d h A::$ Idh $\Delta / / d D:: F R T$ & This study \\
\hline $\mathrm{LA} 20 \Delta / / d D$ & LA02 $\Delta m g s A:: F R T \Delta / d h A:: I d h \quad \Delta / l d D:: F R T$ & This study \\
\hline \multicolumn{3}{|l|}{ Plasmids } \\
\hline pCP20 & reppSC101ts ApR CmR cl857 I PR flp+ & {$[42]$} \\
\hline pZSblank & $\begin{array}{l}\text { Blank plasmid created by removing C. freundii dhaKL from pZSKLcf and self-ligating the plasmid (tetR, oriR } \\
\text { SC101*, cat) }\end{array}$ & {$[11]$} \\
\hline pWM91 & f1(+) ori lacZa of pBluescript II (SK+) mobRP4, oriR6K,SacB and AmpR & [39] \\
\hline pZSKLMgldA & E. coli dhaKLM and gldA under control of PLtetO-1 (tetR, oriR SC101*, cat) & {$[11]$} \\
\hline pZSglpKglpD & E. coli glpK and glpD under control of $\mathrm{P}_{\text {Lteto-1 }}$ (tetR, oriR SC101*, cat) & [15] \\
\hline pZSIdh & S. bovis Idh under control of $\mathrm{P}_{\text {Lteto-1 }}$ (tetR, oriR SC101*, cat) & This study \\
\hline \multicolumn{3}{|l|}{ Primers $^{b}$} \\
\hline$v-p f l B$ & aaatccacttaagaaggtaggtgtcgtggagcctttattgtac & This study \\
\hline $\mathrm{v}-\mathrm{frdA}$ & taccctgaagtacgtggctgaggtagttgcgtcataaggc & This study \\
\hline v-pta & ccaaccaacgaagaactggttagcgcaaatattcccttgc & This study \\
\hline v-adhE & cgagcagatgatttactaaaaaagatcggcattgcccagaagg & This study \\
\hline $\mathrm{v}-1 / d D$ & cagtttcgatattctggaagcgacagattcatgctgcg & This study \\
\hline$v-l d h A$ & gcttaaatgtgattcaacatcactggagaatagaggatgaaaggtcattg & This study \\
\hline$c-l d h$ & gacggtaccatgactgcaactaaacaacacaaaaaaggtacggatccttagtttttgcaagcagaagcgaattc & This study \\
\hline$r 1-l d h$ & tgctgtacatgactgcaactaaacaacactcgtgtacattagtttttgcaagcagaagc & This study \\
\hline r2-ldh & cttacggtcaattgttgacgcgtcaacaattgaccgtaag & This study \\
\hline
\end{tabular}

a Deletions were moved into each strain in the order they appear in the "description" column.

b " $\mathrm{v}$ ", " $\mathrm{c}$ " and " $\mathrm{r}$ " indicate the primer sequences ( $5^{\prime}$ to $\left.3^{\prime}\right)$ that were used for verification purposes (" $\mathrm{v}$ ") during gene disruptions, cloning (" $\mathrm{c}$ ") of $\mathrm{S}$. bovis Idh, and chromosomal replacement (" $\mathrm{r}$ ") of E. coli IdhA with S. bovis Idh (" $\mathrm{r}$ "). " $\mathrm{r} 1$ " and " $\mathrm{r} 2$ " were used to confirm the presence $S$. bovis Idh in the E. coli chromosome (" $\mathrm{r} 1$ ") along wit its presence in the IdhA locus ("r2"). The forward sequence follows the reverse sequence in each case. Genes or operons manipulated are apparent from primer names.

"verification" primers shown in Table 3. The disruption of multiple genes in a common host was achieved as previously described [11]. All resulting strains, along with primers and plasmids used in this study, are listed in Table 3.

Gene overexpression was achieved by cloning the desired gene(s) in a low-copy vector as previously reported [15] (Table 3). Plasmid pZSldh was constructed as follows. The $l d h$ gene from S. bovis was PCR amplified from plasmid pVALDH1 [40] using c-ldh primers (Table 3). The resulting PCR product was cloned within the KpnI and MluI sites of pZSKLMgldA [11] using In-Fusion PCR cloning (Clontech Laboratories, Inc., Mountain View, CA). PCR was performed using Pfu turbo DNA polymerase (Stratagene, CA, USA) under standard conditions described by the supplier. The ligated products were used to transform E. coli DH5 $\alpha \mathrm{T} 1$ (Invitrogen, Carlsbad, CA). Positive clones were screened by plasmid isolation and restriction digestion.

Standard recombinant DNA procedures were used for gene cloning, plasmid isolation, and electroporation. Manufacturer protocols and standard methods [37,43] were followed for DNA purification (Qiagen, Valencia, CA), restriction endonuclease digestion (New England Biolabs, Ipswich, MA), and DNA amplification (Stratagene, La Jolla, CA and Invitrogen, Carlsbad, CA). The strains were kept in $32.5 \%$ glycerol stocks at $-80^{\circ} \mathrm{C}$. Plates were prepared using LB medium containing 1.5\% agar, and appropriate 
antibiotics were included at the following concentrations: ampicillin $(50 \mu \mathrm{g} / \mathrm{ml})$, kanamycin $(50 \mu \mathrm{g} / \mathrm{ml})$, chloramphenicol $(12.5 \mu \mathrm{g} / \mathrm{ml})$, and tetracycline $(3.33 \mu \mathrm{g} / \mathrm{ml})$.

\section{Culture medium and cultivation conditions}

Unless otherwise stated, all fermentations were conducted using the minimal medium designed by Neidhardt et al. [44] with $\mathrm{Na}_{2} \mathrm{HPO}_{4}$ in place of $\mathrm{K}_{2} \mathrm{HPO}_{4}$ and supplemented with $20 \mathrm{~g}$ /liter glycerol (unless otherwise specified), $5 \mu \mathrm{M}$ sodium selenite, $3.96 \mathrm{mM} \mathrm{Na} \mathrm{HPO}_{4}$, $5 \mathrm{mM}\left(\mathrm{NH}_{4}\right)_{2} \mathrm{SO}_{4}$, and $30 \mathrm{mM} \mathrm{NH} \mathrm{ml}_{4}$. Chemicals were obtained from Fisher Scientific (Pittsburgh, PA) and Sigma-Aldrich Co. (St Louis, MO), except crude glycerol, which was provided by Renewable Energy Group, Inc. (Ames, IA). Crude glycerol had the following composition (wt/wt\%): glycerol (83.3), methanol (0.01), water (10.0), fatty acids (0.04), salt (6.63), and ash (6.6). The $\mathrm{pH}$ was 6.38 and the density was $1.26 \mathrm{~g} / \mathrm{ml}$.

Fermentations in shake flasks were performed in $25 \mathrm{ml}$ Pyrex Erlenmeyer flasks (narrow mouth/heavy duty rim, Corning Inc., Corning, NY) filled with $15 \mathrm{ml}$ of $1 \mathrm{X}$ MOPS minimal media supplemented with appropriate antibiotics or inducers when needed at the following concentrations: ampicillin $(50 \mu \mathrm{g} / \mathrm{ml})$, kanamycin $(50 \mu \mathrm{g} / \mathrm{ml})$, chloramphenicol $(12.5 \mu \mathrm{g} / \mathrm{ml})$, tetracycline $(3.33 \mu \mathrm{g} / \mathrm{ml})$, and anhydrotetracycline $(100 \mathrm{ng} / \mathrm{ml})$. Unless otherwise stated, calcium carbonate (5\% wt/wt) was used in all the fermentation flasks to buffer the $\mathrm{pH}$. The flasks (with foam plugs filling the necks) were incubated at $37^{\circ} \mathrm{C}$ and $200 \mathrm{rpm}$ in an NBS C24 Benchtop Incubator Shaker (New Brunswick Scientific Co., Inc., Edison, NJ). The fermentations were run for 36 hours (unless otherwise stated) at which time the supernatant was collected, the $\mathrm{pH}$ measured (UB-10, Denver Instruments Co., Arvada, CO), the optical density taken (Thermo Spectronic Genesys 20, 4001/4, MA, USA), and when necessary cell pellets collected for enzyme activity assays. To determine the optical densities of the cultures in the presence of calcium carbonate, the cultures were allowed to briefly sit in which time the calcium carbonate quickly settled to the bottom.

Prior to use, the cultures (stored as glycerol stocks at $-80^{\circ} \mathrm{C}$ ) were streaked onto LB plates and incubated overnight at $37^{\circ} \mathrm{C}$. Three colonies were used to inoculate 25-ml flasks containing $5 \mathrm{ml}$ of minimal medium supplemented with $10 \mathrm{~g} /$ liter of glycerol, $10 \mathrm{~g} / \mathrm{liter}$ tryptone, and $5 \mathrm{~g} /$ liter yeast extract. The flasks were incubated at $37^{\circ} \mathrm{C}$ and $150 \mathrm{rpm}$ in an NBS C24 Benchtop Incubator Shaker until an $\mathrm{OD}_{550}$ of $\sim 0.7$ was reached. An appropriate volume of this actively growing pre-culture was centrifuged, and the pellet was washed and used to inoculate $15 \mathrm{ml}$ of medium in shake flasks (see above) with a target initial optical density at $550 \mathrm{~nm}$ of 0.05 .

\section{Analytical methods}

The concentration of cell mass, glycerol, organic acids, and ethanol were measured as previously described $[45,46]$. The enantiomeric purity of lactate was determined enzymatically as previously reported [47]. The reaction mixture $(3 \mathrm{ml})$ for L-lactate determination contained $0.92 \mathrm{ml}$ hydrazine/glycine buffer $(0.6 \mathrm{M}$ glycine and $0.5 \mathrm{M}$ hydrazine; pH 9.2), $55 \mathrm{U}$ L-lactate dehydrogenase, $5 \mathrm{mg}$ NAD, and $200 \mu \mathrm{L}$ of the fermentation sample of interest. D-lactate was measured in a similar mixture by replacing L-lactate dehydrogenase with $15 \mathrm{U}$ of $\mathrm{D}$ lactate dehydrogenase. After addition of the sample, the reaction mixture was incubated at $25^{\circ} \mathrm{C}$ for 3 hours after which the absorbance at $340 \mathrm{~nm}$ was used as a measure of the concentration of D- or L-lactate present.

\section{Enzyme activities}

Cell harvesting and preparation of crude cell extracts for enzyme assays was conducted as described elsewhere [5,7]. Absorbance changes for all assays were monitored in a Biomate 5 spectrophotometer (Thermo Scientific, MA, USA). The linearity of reactions (protein concentration and time) was established for all assays and the nonenzymatic rates were subtracted from the observed initial reaction rates. Enzymatic activities are reported as $\mu \mathrm{mol}$ of substrate per minute per mg of cell protein and represent averages for at least three cell preparations. A protein content of $55 \%$ (wt/wt) for E. coli cells was assumed in these calculations.

Glycerol kinase and aerobic-glycerol-3-phosphate dehydrogenase activities were assayed as reported previously [15]. Details of the assay can be found elsewhere [15]. The activity of glycerol dehydrogenase in the oxidation of glycerol was measured as previously described [6] with potassium carbonate at $\mathrm{pH} 9.5$ as the buffer. PEP-dependent dihydroxyacetone kinase activity was assayed as previously reported [11]. D-lactate dehydrogenase activity was determined by following the NADHdependent reduction of pyruvate at $340 \mathrm{~nm}$ and $25^{\circ} \mathrm{C}$ in a $1 \mathrm{ml}$ reaction mixture containing $0.1 \mathrm{M}$ potassium phosphate buffer ( $\mathrm{pH} 7.5), 30 \mathrm{mM}$ sodium pyruvate, $0.33 \mathrm{mM}$ NADH, and $50 \mu \mathrm{L}$ crude cell extract [48]. The activity of L-lactate dehydrogenase (encoded by S. bovis $l d h$ ) was determined as described above for D-lactate dehydrogenase but adding fructose 1,6-bisphosphate, an allosteric activator of $\mathrm{S}$. bovis $\mathrm{L}-\mathrm{LDH}$ [40], to the mixture at a final concentration of $1.2 \mathrm{mM}$.

\section{Calculation of fermentation parameters}

Data from cell growth, glycerol consumption, and product synthesis were used to calculate volumetric (g/liter/h) and specific rates (g/g cell mass/h) and product yields $(\mathrm{g} / \mathrm{g}$ glycerol) as previously described $[5,11]$. 


\section{Additional file}

Additional file 1: Figure S1. Percent dissolved oxygen (DO) and oxygen transfer rate (OTR) vs. time of wild-type and the final L-lactate biocatalyst. Symbols denote: \% DO (घ) and OTR ( $\mathbf{\Delta})$. (A) Wild-type MG1655. (B) LA20 /ldD [pZS-glpK-glpD].

\section{Competing interests}

The authors declare that they have no competing interests.

\section{Authors' contributions}

RG conceived the study. SM, JMC and MDB conducted the experiments. RG, SM, MDB, AM and JMC analyzed the data and prepare the manuscript. All authors read and approved the final manuscript.

\section{Acknowledgements}

This work was supported by grants from the U.S. National Science Foundation (CBET-0645188) and the National Research Initiative of the U.S. Department of Agriculture Cooperative State Research, Education and Extension Service (2005-35504-16698). We thank H. Mori and T. R. Whitehead for providing research materials, C. Rivera, S. Doneske, and S. S. Yazdani for assistance with genetic methods, Alfredo Martinez Jimenez for assistance in the quantification of oxygen transfer, and Paul Campbell for fruitful discussions.

Received: 27 September 2012 Accepted: 20 January 2013 Published: 25 January 2013

\section{References}

1. Khanal SK, Rasmussen M, Shrestha P, Van Leeuwen H, Visvanathan C, Liu H: Bioenergy and biofuel production from wastes/residues of emerging biofuel industries. Water Environ Res 2008, 80:1625-1647

2. Rausch KD, Belyea RL: The future of co-products from corn processing. Appl Biochem Biotechnol 2006, 128:47-86.

3. Oren A: A hundred years of Dunaliella research: 1905-2005. Saline Syst 2005, 1:2.

4. Clomburg JM, Gonzalez R: Biofuel production in Escherichia coli: the role of metabolic engineering and synthetic biology. App Microbiol Biotechnol 2010, 86:419-434.

5. Durnin G, Clomburg J, Yeates Z, Alvarez PJJ, Zygourakis K, Campbell P, Gonzalez R: Understanding and harnessing the microaerobic metabolism of glycerol in Escherichia coli. Biotechnol Bioeng 2009, 103:148-161.

6. Gonzalez R, Murarka A, Dharmadi Y, Yazdani SS: A new model for the anaerobic fermentation of glycerol in enteric bacteria: trunk and auxiliary pathways in Escherichia coli. Metab Eng 2008, 10:234-245.

7. Murarka A, Dharmadi Y, Yazdani SS, Gonzalez R: Fermentative utilization of glycerol in Escherichia coli and its implications for the production of fuels and chemicals. Appl Environ Microbiol 2008, 74:1124-1135.

8. Gonzalez R, Campbell P, Wong M: Production of ethanol from thin stillage by metabolically engineered Escherichia coli. Biotechnol Lett 2010, 32:405-411.

9. Nikel PI, Ramirez MC, Pettinari MJ, Méndez BS, Galvagno MA: Ethanol synthesis from glycerol by Escherichia coli redox mutants expressing adhE from Leuconostoc mesenteroides. J Appl Microbiol 2010, 109:492-504

10. Trinh CT, Srienc F: Metabolic engineering of Escherichia coli for efficient conversion of glycerol to ethanol. Appl Environ Microbiol 2009, 75:6696-6705.

11. Yazdani SS, Gonzalez R: Engineering Escherichia coli for the efficient conversion of glycerol to ethanol and co-products. Metab Eng 2008 10:340-351.

12. Hu HB, Wood TK: An evolved Escherichia coli strain for producing hydrogen and ethanol from glycerol. Biochem Biophys Res Comm 2010, 391:1033-1038.

13. Zhu YH, Eiteman MA, Lee SA, Altman A: Conversion of glycerol to pyruvate by Escherichia coli using acetate and acetate/glucose-limited fed-batch processes. J Ind Microbiol Biotechnol 2010, 37:307-312.

14. Zhang XL, Shanmugam KT, Ingram LO: Fermentation of glycerol to succinate by metabolically engineered strains of Escherichia coli. Appl Environ Microbiol 2010, 76:2397-2401.
15. Mazumdar S, Clomburg JM, Gonzalez R: Engineered Escherichia coli strains for the homofermentative production of D-lactic acid from glycerol. Appl Environ Microbiol 2010, 76:4327-4336

16. Hofvendahl K, Hahn-Hagerdal B: Factors affecting the fermentative lactic acid production from renewable resources. Enzyme Microb Technol 2000, 26:87-107.

17. Okano K, Tanaka T, Ogino C, Fukuda H, Kondo A: Biotechnological production of enantiomeric pure lactic acid from renewable resources: recent achievements, perspectives, and limits. App/ Microbio/ Biotechnol 2010, 85:413-423.

18. Kharas GB, Sanchez-Riera F, Severson DK: Polymers of lactic acid. In Plastics from microbes: microbial synthesis of polymers and polymer precursors. Edited by Mobley DP. Munich, Germany: Hanser Publishers; 1994

19. Vaidya AN, Pandey RA, Mudliar S, Kumar MS, Chakrabarti T, Devotta S: Production and recovery of lactic acid for polylactide-an overview. Crit Rev Environ Sci Technol 2005, 35:429-467.

20. Chang DE, Jung HC, Rhee JS, Pan JG: Homofermentative production of D- or L-lactate in metabolically engineered Escherichia coli RR1. Appl Environ Microbiol 1999, 65:1384-1389.

21. Dien BS, Nichols NN, Bothast RJ: Recombinant Escherichia coli engineered for production of L-lactic acid from hexose and pentose sugars. J Ind Microbiol Biotechnol 2001, 27:259-264.

22. Zhou S, Shanmugam KT, Ingram LO: Functional replacement of the Escherichia coli $\mathrm{D}(-)$-lactate dehydrogenase gene (IdhA) with the $\mathrm{L}$ (+)-lactate dehydrogenase gene (ldhL) from Pediococcus acidilactici. Appl Environ Microbiol 2003, 69:2237-2244.

23. Zhu Y, Eiteman MA, DeWitt K, Altman E: Homolactate fermentation by metabolically engineered Escherichia coli strains. Appl Environ Microbio 2007, 73:456-464.

24. Baldoma L, Aguilar J: Involvement of lactaldehyde dehydrogenase in several metabolic pathways of Escherichia coli K12. J Biol Chem 1987 262:13991-13996.

25. Booth IR, Ferguson GP, Miller S, Li C, Gunasekera B, Kinghorn S: Bacterial production of methylglyoxal: a survival strategy or death by misadventure? Biochem Soc Trans 2003, 31:1406-1408.

26. Booth IR: Glycerol and methylglyoxal metabolism. In EcoSal-Escherichia coli and Salmonella: cellular and molecular biology. Edited by Curtis R III. Washington, D.C: ASM press; 2005.

27. Totemeyer S, Booth NA, Nichols WW, Dunbar B, Booth IR: From famine to feast: the role of methylglyoxal production in Escherichia coli. Mol Microbiol 1998, 27:553-562.

28. Schweizer H, Larson TJ: Cloning and characterization of the aerobic snglycerol-3-phosphate dehydrogenase structural gene glpD of Escherichia coli K-12. J Bacteriol 1987, 169:507-513.

29. Walz AC, Demel RA, de Kruijff B, Mutzel R: Aerobic sn-glycerol-3-phosphate dehydrogenase from Escherichia coli binds to the cytoplasmic membrane through an amphipathic alpha-helix. Biochem J 2002, 365:471-479.

30. Iuchi S, Aristarkhov A, Dong JM, Taylor JS, Lin EC: Effects of nitrate respiration on expression of the Arc-controlled operons encoding succinate dehydrogenase and flavin-linked L-lactate dehydrogenase. J Bacteriol 1994, 176:1695-1701

31. Yazdani SS, Gonzalez R: Anaerobic fermentation of glycerol: a path to economic viability for the biofuels industry. Curr Opin Biotechnol 2007, 18:213-219.

32. Unden G, Dünnwald P: The aerobic and anaerobic respiratory chain of Escherichia coli and Salmonella enterica: enzymes and energetics. In EcoSal_Escherichia coli and Salmonella: cellular and molecular biology. Edited by Curtis R III. Washington, D.C: ASM press; 2008

33. Kashket ER: Stoichiometry of the H+-ATPase of Escherichia coli cells during anaerobic growth. FEBS Lett 1983, 154:343-346.

34. Jantama K, Haupt MJ, Svoronos SA, Zhang XL, Moore JC, Shanmugam KT, Ingram LO: Combining metabolic engineering and metabolic evolution to develop non recombinant strains of Escherichia coli $\mathrm{C}$ that produce succinate and malate. Biotechnol Bioeng 2008, 99:1140-1153.

35. Yomano LP, York SW, Zhou S, Shanmugam KT, Ingram LO: Re-engineering Escherichia coli for ethanol production. Biotechnol Lett 2008, 30:2097-2103.

36. Zhang X, Jantama K, Moore JC, Shanmugam KT, Ingram LO: Production of L-alanine by metabolically engineered Escherichia coli. App/ Microbiol Biotechnol 2007, 77:355-366. 
37. Miller JH: Experiments in Molecular Genetics. New York: Cold Spring Harbor Laboratory Press; 1972.

38. Baba T, Ara T, Hasegawa M, Takai Y, Okumura Y, Baba M, Datsenko KA, Tomita M, Wanner BL, Mori H: Construction of Escherichia coli K-12 inframe, single-gene knockout mutants: the Keio collection. Mol Syst Biol 2006, 2:8-18.

39. Metcalf W, Jiang W, Daniels LL, Kim S, Haldimann A, Wanner BL: Conditionally replicative and conjugative plasmids carrying lacZa for cloning, mutagenesis, and allele replacement in bacteria. Plasmid 1996, 35:1-13.

40. Wyckoff HA, Chow J, Whitehead TR, Cotta MA: Cloning, sequence, and expression of the L-(+) lactate dehydrogenase of Streptococcus bovis. Curr Microbiol 1997, 34:367-373.

41. Kang Y, Durfee T, Glasner JD, Qiu Y, Frisch D, Winterberg KM, Blattner FR: Systematic mutagenesis of the Escherichia coli genome. J Bacteriol 2004, 186:4921-4930.

42. Datsenko KA, Wanner BL: One-step inactivation of chromosomal genes in Escherichia coli K-12 using PCR products. Proc Natl Acad Sci USA 2000, 97:6640-6645

43. Sambrook J, Fritsch EF, Maniatis T: Molecular cloning: a laboratory manual. New York: Cold Spring Harbor Laboratory Press; 1989.

44. Neidhardt FC, Bloch PL, Smith DF: Culture medium for enterobacteria. J Bacteriol 1974, 119:736-747.

45. Dharmadi Y, Gonzalez R: A better global resolution function and a novel iterative stochastic search method for optimization of high-performance liquid chromatographic separation. J Chromatogr A 2005, 1070:89-101.

46. Dharmadi Y, Murarka A, Gonzalez R: Anaerobic fermentation of glycerol by Escherichia coli: a new platform for metabolic engineering. Biotechnol Bioeng 2006, 94:821-829.

47. Severn DJ, Johnson ME, Olson NF: Determination of lactic acid in Cheddar cheese and calcium lactate crystals. J Dairy Sci 1986, 69:2027-2030

48. Tarmy EM, Kaplan NO: Kinetics of Escherichia coli B D-lactate dehydrogenase and evidence for pyruvate controlled change in conformation. J Biol Chem 1968, 243:2587-2596.

doi:10.1186/1475-2859-12-7

Cite this article as: Mazumdar et al:: Efficient synthesis of L-lactic acid from glycerol by metabolically engineered Escherichia coli. Microbial Cell Factories 2013 12:7.

\section{Submit your next manuscript to BioMed Central and take full advantage of:}

- Convenient online submission

- Thorough peer review

- No space constraints or color figure charges

- Immediate publication on acceptance

- Inclusion in PubMed, CAS, Scopus and Google Scholar

- Research which is freely available for redistribution 